\title{
Banho enrolado em bebês prematuros em unidade neonatal: a prática na perspectiva de enfermeiros
}

\author{
Swaddle bathing in premature babies in a neonatal unit: the practice from the perspective of \\ nurses
}

\section{Como citar este artigo:}

Santos HM, Silva LJ, Góes FGB, Santos ACN, Araújo BBM, Santos IMM. Swaddle bathing in premature babies in a neonatal unit: the practice from the perspective of nurses. Rev Rene. 2020;21:e42454. DOI: https://doi.org/10.15253/2175-6783.20202142454

\author{
(1)Hisabela Marinheiro dos Santos ${ }^{1}$ \\ (D)Laura Johanson da Silva ${ }^{1}$ \\ (1) Fernanda Garcia Bezerra Góes ${ }^{2}$ \\ (D)Ana Carolina Nascimento dos Santos ${ }^{1}$ \\ DBárbara Bertolossi Marta de Araújo ${ }^{3}$ \\ (DInês Maria Meneses dos Santos ${ }^{1}$
}

\author{
${ }^{1}$ Universidade Federal do Estado do Rio de Janeiro. \\ Rio de Janeiro, RJ, Brasil. \\ ${ }^{2}$ Universidade Federal Fluminense. \\ Rio das Ostras, RJ, Brasil. \\ ${ }^{3}$ Universidade do Estado do Rio de Janeiro. \\ Rio de Janeiro, RJ, Brasil.
}

\author{
Autor correspondente: \\ Laura Johanson da Silva \\ Rua Dr. Xavier Sigaud, 290 - Urca \\ CEP: 22290-180. Rio de Janeiro, RJ, Brasil. \\ E-mail: lauraenfaunirio@gmail.com
}

\begin{abstract}
RESUMO
Objetivo: compreender a prática do banho enrolado em bebês prematuros, em unidade neonatal, na perspectiva de enfermeiros. Métodos: pesquisa qualitativa, realizada por meio de entrevista semiestruturada, com 13 enfermeiros atuantes em unidades neonatais, captados através da técnica de amostragem bola de neve. Dados submetidos à análise temático-categorial. Resultados: os enfermeiros consideraram benefícios relacionados ao conforto e à estimulação no banho enrolado, entretanto, citaram desafios para a prática, relacionados ao desconhecimento, à baixa adesão e sobrecarga de trabalho da equipe de enfermagem, ausência de rotinas e protocolos institucionais, além da escassez ou mesmo inadequação dos recursos materiais disponíveis nas instituições. Conclusão: a prática do banho enrolado, na perspectiva de enfermeiros, tem efeitos positivos para o desenvolvimento de bebês prematuros, porém existem desafios gerenciais que precisam ser superados para efetiva implementação.

Descritores: Enfermagem; Recém-Nascido Prematuro; Humanização da Assistência; Unidades de Terapia Intensiva Neonatal; Imersão.
\end{abstract}

\begin{abstract}
Objective: to understand the practice of swaddle bathing in premature babies, in a neonatal unit, from the perspective of nurses. Methods: qualitative research, conducted through semi-structured interview, with 13 nurses working in neonatal units, captured through the snowball sampling technique. Data submitted to thematic-categorical analysis. Results: nurses considered benefits related to comfort and stimulation in the swaddle bathing. However, they mentioned challenges for practice, related to lack of knowledge, low adherence and work overload of the nursing team, absence of routines and institutional protocols, besides to scarcity or even inadequacy of the material resources available in the institutions. Conclusion: the practice of swaddle bathing, from the perspective of nurses, has positive effects for the premature babies' development. Nevertheless, there are managerial challenges that need to be overcome for effective implementation.

Descriptors: Nursing; Infant, Premature; Humanization of Assistance; Intensive Care Units, Neonatal; Immersion.
\end{abstract}




\section{Introdução}

A mortalidade infantil no Brasil tem, atualmente, o período neonatal como principal componente etário, sendo a prematuridade causa preponderante de óbitos ${ }^{(1)}$. Para além da desafiadora redução da morbimortalidade entres bebês de risco, o objetivo da assistência neonatal envolve a redução de danos para o melhor desenvolvimento infantil da população de prematuros $^{(2)}$.

Tendo que se adaptar ao ambiente da unidade neonatal, bebês prematuros enfrentam considerável falta de energia e capacidades fisiológicas adaptativas, limitadas pela imaturidade de seus sistemas. Nesse contexto, a manipulação excessiva, associada a estímulos nocivos que provocam dor e estresse, possui impacto negativo no desenvolvimento cerebral, por isso, a premente necessidade de cuidados que sejam individualizados, neuroprotetores e permitam a organização fisiológica e comportamental. Esse tipo de modulação é proposto pelo Newborn Individualized Developmental Care and Assessment Program (NIDCAP), cuja lógica foi incorporada no Brasil, dentro do escopo das ações de humanização da assistência neonatal, pelo Método Canguru ${ }^{(3-5)}$.

Entre os cuidados de enfermagem em unidades neonatais que envolve alta manipulação e que tem sido alvo de pesquisas quanto ao impacto na regulação fisiológica e comportamental do prematuro está o banho, com repercussões para os parâmetros de frequência cardíaca, saturação de oxigênio e temperatura, além de tempo de choro e sinais de estresse $\mathrm{e}^{(6-7)}$.

$\mathrm{Na}$ perspectiva dos cuidados desenvolvimentais, o banho de imersão, proposto pelo Ministério da Saúde do Brasil, é conhecido em nível nacional como banho humanizado ${ }^{(3)}$ e abordado na literatura internacional como banho enrolado ${ }^{(7-9)}$, prática que consiste na imersão do bebê em água morna até logo abaixo dos ombros, envolvido em tecido, mantendo braços e pernas em flexão. 0 processo de desdobramento do tecido, higienização e retorno do tecido é feito, cuidadosamente, em partes, a fim de promover contenção, resultando em redução do estresse comportamental, devido a maior estabilidade dos sistemas autonômico e motor, durante e após o procedimento ${ }^{(7-9)}$.

Na prática assistencial, a técnica convencional do banho com a imersão direta do bebê em banheira ou cúpula acrílica é mais conhecida e rotineiramente adotada nas unidades de internação neonatal ${ }^{(6)}$. Por outro lado, a técnica do banho de imersão enrolado precisa ser alvo de investigações científicas para subsidiar mudanças na prática clínica que promovam melhores respostas fisiológicas e comportamentais do bebê prematuro frente a esse cuidado. Em revisão sistemática recente, destacou-se que há escassez de ensaios clínicos centrados no banho enrolado, entretanto, com base nos achados disponíveis, o mesmo foi recomendado, tendo em vista a redução de estresse. Sinalizou-se, também, a importância de apoiar o comportamento protetor do enfermeiro, durante o banho do bebê prematuro, e o treinamento para aquisição de atitudes que minimizem o estresse do bebê $\hat{e}^{(7)}$.

Nessa vertente, a questão de pesquisa foi: como enfermeiros percebem a prática do banho enrolado em bebês prematuros, no contexto da unidade neonatal? Assim, a pesquisa objetivou compreender a prática do banho enrolado em bebês prematuros, em unidade neonatal, na perspectiva de enfermeiros.

\section{Métodos}

Trata-se de estudo qualitativo, no qual se utilizou da técnica bola de neve, para captação dos participantes, a qual consiste em forma de amostragem não probabilística, por meio de cadeias de referência para localizar possíveis participantes, sendo utilizada, principalmente, para fins exploratórios e para pesquisar grupos difíceis de serem acessados ${ }^{(10)}$. A adoção desta estratégia neste estudo ocorreu, especialmente, pela dificuldade de localizar potencial cenário cuja prática do banho enrolado em prematuros integrasse a rotina assistencial.

O cenário de opção para captação dos informantes-chave ("sementes") foi o Núcleo de Pesquisa, 
Experimentação e Estudos em Enfermagem na Área da Mulher e da Criança, localizado na Escola de Enfermagem Alfredo Pinto, da Universidade Federal do Estado do Rio de Janeiro, pelo fato deste núcleo reunir enfermeiros com experiência em unidade neonatal de instituições públicas e privadas e estudantes de pós-graduação com temas na área da prematuridade. A escolha deste entorno se deu pela facilidade de acesso a informantes-chave com potencial rede de contatos. Os contatos para captação foram realizados por meio telefônico, a partir das indicações sequenciais. As sementes foram indicando um ou mais indivíduos que, quando contactados e agregados aos participantes, indicaram outros e assim por diante, aumentando o quadro de amostragem ${ }^{(10)}$.

Nesse processo, contactaram-se 13 enfermeiros que preencheram os critérios de inclusão: enfermeiros assistenciais com mais de um ano de experiência em unidade neonatal, com que conheciam e desenvolviam a técnica do banho enrolado em bebê prematuro. Não houve recusa ou desistência e, portanto, os 13 enfermeiros compuseram a amostra. A coleta de dados ocorreu de agosto a novembro de 2018, com a utilização de entrevistas semiestruturadas individuais, previamente agendadas, em local reservado, com gravação de voz por mídia digital. 0 roteiro de entrevista foi composto de breve caracterização do participante, contendo as variáveis idade, sexo, tempo de formação, tempo de trabalho na área neonatal, cargo/função e se possuía treinamento teórico ou prático em cuidados desenvolvimentais. Em seguida, procederam-se às perguntas: para você, quais os significados do banho do bebê prematuro em Unidade Neonatal? Que tipo de banho é mais comum na prática diária? Como você desenvolve o banho enrolado e como se sente na interação com o bebê? 0 que você acha desse tipo de banho? Como você conheceu essa técnica? Em seu local de trabalho, há alguma normatização escrita sobre o banho do prematuro? 0 que você julga que seria importante para melhorar o cuidado de enfermagem no banho do prematuro na Unidade Neonatal?

As entrevistas foram conduzidas pela autora principal, mediante treinamento prévio e supervisão da segunda autora, pesquisadora com expertise nesse tipo de pesquisa qualitativa. 0 encerramento do trabalho de campo aconteceu através do critério de saturação dos dados, quando, mediante análise, não se observou entrada de novas informações para esclarecimento do objeto estudado. As entrevistas tiveram duração média de 15 minutos.

Após transcrição, os dados foram submetidos à análise de conteúdo temático-categorial que apresenta as seguintes etapas: pré-análise, exploração do material ou codificação, tratamento dos resultados-inferência e interpretação ${ }^{(11)}$. Nessas etapas, as unidades de significação foram organizadas em quadros analíticos, sendo contabilizada a recorrência em termos de unidades de registro dentro de cada entrevista transcrita e entre elas, buscando-se, também, a aderência temática para categorização. Desse processo, emergiram três categorias temáticas: Benefícios da técnica do banho enrolado em unidade neonatal; Indicações e práticas do banho enrolado em unidade neonatal; Desafios gerenciais para prática do banho em Unidade Neonatal.

Em relação aos aspectos éticos, a pesquisa possui o Certificado de Apresentação para Apreciação Ética, conforme número 91292718.2.0000.5285, e aprovação no Comitê de Ética da Universidade Federal do Estado do Rio de Janeiro, parecer de número 2.769.369/2018, segundo Resolução no 466/2012, do Conselho Nacional de Saúde. Aos participantes, solicitaram-se consentimento prévio, mediante informações sobre a pesquisa, e assinatura do Termo de Consentimento Livre e Esclarecido. Para garantir o sigilo dos participantes, os depoimentos foram identificados no estudo através do código "E" para Enfermeiros, com a sequência numérica de entrada na pesquisa (exemplo: E1, E2).

\section{Resultados}

Os participantes deste estudo (13) eram do sexo feminino, com idades entre 26 e 58 anos. 0 tempo de 
formação profissional variou de dois a 34 anos, com média de 14,2 anos. Em relação ao tempo de trabalho em unidade neonatal, este variou de um ano e 10 meses a 34 anos, com média de 12,6 anos de experiência entre os participantes. Somente uma enfermeira era de hospital da rede privada, as demais (12) eram de hospitais e maternidades públicas da cidade do Rio de Janeiro, Brasil, sendo oito enfermeiras da rede federal, uma da rede estadual e três da rede municipal. Todos relataram possuir treinamento teórico ou prático em cuidados desenvolvimentais. Somente duas (2) enfermeiras afirmaram que o banho enrolado constava nos protocolos e na prática assistencial nas unidades que atuavam.

\section{Benefícios da técnica do banho enrolado em uni- dade neonatal}

Os enfermeiros mencionaram que o banho enrolado tem benefícios para o bebê relacionados ao relaxamento e menor desorganização comportamental, com reflexos para o crescimento e desenvolvimento do bebê, por evitar intercorrências, como estresse, choro, perda de peso, queda de saturação e apneia. $A$ gente consegue trazer benefícios para esse crescimento do bebê, o bebê não fica estressado, se ele não chora muito, ele não perde peso, se ele consegue relaxar, ele consegue manter todos os seus parâmetros hemodinâmicos normais e isso ajuda no crescimento e no próprio desenvolvimento daquele bebê. Não tem queda de saturação, não tem apneia na hora do banho, não tem nenhuma intercorrência, você consegue dar aquele banho tranquilamente, sem intercorrência no bebê (E2). O profissional se sente grato em ver que a criança fica menos desorganizada, fica com a sua parte hemodinâmica mais estável, que a interação e o sono dela depois melhora. Ela tem um sono muito mais duradouro, melhora o ganho de peso (E4).

Os enfermeiros pontuaram que o banho enrolado proporciona ao bebê sensação de segurança, pois imita o que é o meio uterino, através da contenção corporal que o tecido possibilita. A leitura da mímica facial foi importante indicativo apontado, para que o enfermeiro compreenda a reação do bebê durante o cuidado. Porque quando o bebê está intraútero, ele está em um ambiente, em um líquido quente e ele tem ao lado dele o útero e ele não fica tão largado, ele tem uma delimitação ali dentro. E quando a gente coloca o bebê para tomar banho, e o banho é enrolado, a gente consegue que esse bebê entre em contado com a água, uma água morna, e ele enroladinho, ele também vai ter aquela delimitação de espaço (E2). Você vê a carinha de entrega e satisfação do bebê. Ele, às vezes, está ali tomando banho, mas está dormindo profundamente, ele não faz expressão facial nenhuma de dor, então, assim, é um procedimento até amoroso de se ver (E6).

Destacaram, ainda, ser uma prática prazerosa para o profissional, sendo oportunidade de interação e contato com o bebê, tendo em vista que, por vezes, a mãe pode não estar presente. Uma forma de interação, de contato. Porque quando a mãe não está aqui, muitas mães não conseguem ficar aqui diariamente, então, esse bebê acaba tendo pouco contato humano, poucos estímulos. Então, isso é bom também, porque você abre uma janela de interação com o bebê (E9). Para mim, é perfeito. A gente consegue tornar a prática em algo prazeroso para o profissional (E2).

\section{Indicações e práticas do banho enrolado em uni- dade neonatal}

Os enfermeiros reconheceram que a aplicação do banho seguia algumas indicações, como higiene corporal do bebê prematuro, ademais da necessidade de relaxamento, conforto e estimulação sensoriomotora. Contudo, destacaram a importância da avaliação clínica para tomada de decisão. Em um bebezinho prematuro, a gente dá mais como um fator de relaxamento para ele. Então, a gente vê, às vezes, aquele bebezinho que não está com ventilação mecânica, está estável e até bebezinho, às vezes, que está na incubadora e a gente percebe que ele tem toda uma condição adequada para o banho, a gente tira (referindo-se à incubadora) (E1). O prematuro aqui a gente dá o banho, dependendo do peso dele, nem todos os prematuros tomam banho. Mas, esses prematuros maiores, que a gente consegue promover o banho, é importante porque também, além do conforto, ele promove também, eu acho até uma percepção sensoriomotora, sai fora da incubadora e amplia a visão desse prematuro (E12).

Em relação à prática procedimental do banho enrolado, os enfermeiros enfatizaram os cuidados re- 
lacionados ao processo de manejo do bebê, respeito aos estados comportamentais e preocupações com o ambiente do cuidado, especialmente no que se refere à termorregulação e prevenção de infecção. Prepara $a$ água, morninha, depois enrola o bebê, leva até a banheira, inicialmente, ele enroladinho, faz a higiene da face e cabeça e depois a gente coloca ele dentro da banheira com ele enroladinho, faz adaptação nele, e depois, aos poucos, vai tirando o paninho para fazer a higiene que precisa (E13). Eu acho que a pessoa tem que se ajustar ao bebê para fazer esse banho, para que ele dê mais certo, porque se você pega o bebê que estava dormindo e enfia ele na água, ele vai gritar, espernear que nem um louco (E7). A rotina é desligar o ar condicionado lá da unidade central. Esse nosso setor é muito frio. A gente tem na verdade uma banheira própria para poder fazer o banho e a gente faz desinfecção da banheira, a gente tem um termômetro para poder avaliar a temperatura da água, a gente envolve o bebê. Quando a mãe tem uma fralda (referindo-se à fralda de tecido), a gente usa a própria fralda da mãe, porque a gente acha um tecido mais fácil de você contornar o bebê (E1).

Apontaram a priorização da realização do banho enrolado para os bebês prematuros (por serem mais suscetíveis ao estresse) que passam a ser aptos ao banho de imersão, no caso dos bebês a termo, o banho tradicional é o eleito. No entanto, para além da questão de maturidade gestacional, os enfermeiros também salientaram o estresse do bebê como indicação ou mesmo o desejo do profissional como fator que influencia a realização do banho enrolado. Mas, já realize o banho enrolado em bebês prematuros, principalmente em prematuros, porque ele tem um estímulo maior, eles se assustam mais fácil, uma pele mais frágil, uma integridade menor, então o banho enrolado é mais visado para essas crianças menores (E9). Enrolado é o que a gente raramente faz, mas é o mais correto, mas em bebê prematuro com baixo peso, quando ele pode tomar banho ele faz sim. Bebês a termo, não. Bebês prematuros com estabilidade se realiza, mas os a termo, não. A termo, colocamos direto na banheira mesmo (E10). A gente acaba usando essa técnica quando o bebê é mais estressado e acaba você escolhendo os bebês que você acha que deve fazer diferente ou quando você estiver a fim (E1).

Os enfermeiros consideraram importante a presença de um familiar nesse momento, participando de forma ativa, de modo a promover segurança pa- rental e chances de continuidade do cuidado, no processo de transição do hospital para casa. Como o banho é realizado durante o dia, os pais estão perto, então você acaba passando certa segurança para os pais, porque ele vê o bebê calmo, relaxado durante o banho. Com a técnica do enrolamento, da humanização, a gente consegue ter essa interação bem gostosa, bem satisfatória e proporcionar conforto para o bebê e conseguir ter interação até mesmo familiar e ver também que a gente acaba incentivando que os pais também façam o mesmo procedimento, tanto na unidade ou até mesmo após a alta e isso também ajuda a gerar certa segurança nos pais (E2). É importante, se possível, sempre chamar a mãe. A gente cuida do bebê para ele ir para casa, então que a mãe estivesse junto para observar, para ela não se surpreender no dia da alta e ficar aquela correria para aprender como é que é o banho ou em casa. Já que o bebê está aqui, ela já ir aprendendo a fazer esse banho. E isso é importante, é relaxante, e inserir a família é tão importante quanto (E13).

\section{Desafios gerenciais para prática do banho em Uni- dade Neonatal}

Apesar dos benefícios fisiológicos e comportamentais que o banho enrolado proporciona para o bebê prematuro, os enfermeiros afirmaram que observam pouca utilização dessa técnica na equipe e que as instituições em que atuam, ainda, mantêm como protocolo a prática do banho tradicional de imersão, sem enrolamento, para bebês aptos a sair da incubadora. Somente dois enfermeiros afirmaram realizar o banho enrolado como rotina institucionalizada, por existência de protocolo na unidade. Houve menção também do uso do enrolamento em banho de ofurô, que se trata de imersão em balde, pelo profissional de fisioterapia, com fins terapêuticos. $O$ mais comum éo banho convencional, o nosso protocolo da unidade é o banho convencional, mas a gente está começando a alterar o protocolo para o banho humanizado (E1). A gente escuta muito falar do banho enrolado e tal, mas assim, eu vejo pouco na minha unidade a equipe técnica fazendo a técnica do banho enrolado (E6). É o banho enrolado, tanto os prematuros que atingem a idade gestacional quanto os bebês a termo (E11). Ele indica o banho enrolado (referindo-se ao protocolo). Ele estabelece dias para o banho e diz que tem que ser com o mínimo manuseio e o mínimo invasivo possível. Temos a prática de fazer o banho 
enrolado em prematuros (E9). Se o paciente já estivesse no peito da mãe ou no berçário, quase saindo de alta, a gente dava o banho de imersão que era o banho no ofurô, mas não era dado pela enfermagem, era dado pela fisioterapeuta (E3).

Outro motivo para não aplicabilidade do banho enrolado é a relação de quantitativo de profissionais por paciente, o que afeta a dinâmica de trabalho, logo, essa prática, considerada também como terapêutica, acaba ficando restrita a alguns bebês selecionados. Aqui, o mais comum é o tradicional, porque têm muitos leitos, e sempre fica além da capacidade, são poucos profissionais e crianças de alto risco. Então, acaba que a prática do banho é o mais tradicional, por questões de higiene, quando a criança necessita é o banho terapêutico, ele é mais direcionado para alguns casos mais específicos (E9).

Além disso, foi relatado pelos enfermeiros como motivos para não realização do banho enrolado a dinâmica da própria equipe, carência de alguns insumos materiais, resistência por parte dos profissionais ou escassez de treinamento. Muito melhor você dar banho em um bebê mais tranquilo do que você, durante o procedimento, o bebê ficar todo desorganizado e chorando. Mas, nem sempre se consegue, por conta de rotina mesmo, das pessoas, da unidade, e falta algum dispositivo que vá te ajudar (E5). Têm alguns fatores que interferem e que dificultam, às vezes, o banho, tipo a falta de lençol. A falta de lençol é o principal, nem sempre a gente tem para fazer o banho, para secar a criança e para ficar na caminha, o principal mesmo é a falta de insumos. E tudo que é uma técnica nova, tem um pouco de bloqueio com aqueles que são mais antigos, mas a partir do momento que você explica os benefícios do banho mais humanizado, eles vão se adaptando à técnica, mas é com o tempo, nada imediato (E10). Na técnica, mesmo do banho enrolado, você coloca o bebê na água enroladinho e vai tirando os panos aos pouquinhos, eu, geralmente, não faço isso, eu realmente tiro o pano e coloco na água. Mas, isso eu acho que é treino mesmo, e como a gente acaba não fazendo a técnica, não temos o treino disso corretamente (E6).

Os enfermeiros consideraram relevante estabelecer o banho enrolado como rotina na instituição, através de sistematização, em forma de procedimento operacional padrão, uma vez que a realização do procedimento, ainda, é centrada na opção e no desejo do profissional. Eu acho o melhor para o bebê, então tanto é que a gente está fazendo a revisão dos POP (referindo-se ao procedimento operacional padrão), agora, e a gente quer introduzir como uma rotina na unidade, e não assim: 'Eu estou a fim, eu faço!', entendeu!? A gente quer botar como uma rotina da unidade. Ultimamente, somente quem quer que faz, porque o POP da unidade é o banho tradicional (E1).

Os entrevistados expressaram necessidade de conscientização, conhecimento e capacitação da equipe sobre as mudanças e os benefícios do banho enrolado em prematuros, para conseguir maior aceitação e engajamento dos profissionais para realização dessa técnica, e desenvolvimento de olhar clínico diante das situações. Eu acho que é a conscientização e a capacitação de todos os profissionais em conjunto, a gente vai conseguir com que todos tenham o verdadeiro entendimento e saiba o real objetivo, então, a gente consegue ter um compromisso maior, por parte de todos da equipe (E2). Eu acho que é a sensibilização da equipe, é você ter principalmente uma educação continuada, porque aqui a gente não tem, porque, muitas vezes, é o técnico que está diretamente ligado a esses cuidados com o bebê e não que eles não saibam, mas muitos não têm conhecimento. Não tiveram acesso a isso, então, eles desconhecem, então, às vezes fazem ou deixam de fazer alguma coisa, por falta de ter tido conhecimento. Então, é importante ter um profissional que venha e faça uma educação continuada (E8).

\section{Discussão}

Por ser ainda prática pouco sistematizada em termos protocolares em instituições locais, as limitações dos resultados se referem à regionalidade e dificuldade de aprofundamento contextual, por ter sido realizado com participantes de diferentes unidades neonatais. Apesar disto, essa diversidade possibilitou destacar importantes aspectos assistenciais e gerenciais que influenciam a prática do banho em unidade neonatal.

Dessa forma, o reconhecimento dos efeitos positivos do banho enrolado, evidenciados no estudo, contribui para disseminação e fortalecimento dessa técnica, na prática clínica, com sustentação teórica. Por outro lado, a identificação das dificuldades para implementação pode balizar o desenvolvimento de ações gerenciais que promovam a devida aplicabilida- 
de, como atualizações e treinamento sobre a temática junto aos profissionais de enfermagem, criação de rotinas e protocolos institucionais para padronização da técnica e aquisição de materiais próprios para realização do procedimento.

Nessa diretiva, os resultados deste estudo apontaram que os enfermeiros participantes compreendiam o impacto do manuseio no banho sobre a estabilidade fisiológica e comportamental do bebê e reconheciam, na prática do banho enrolado, oportunidades para promover conforto e a estimulação neuroprotetor. Tais achados corroboram com evidências de revisão sistemática, em que esse tipo de banho demonstrou ser o melhor método para prematuros, em unidade de terapia intensiva neonatal, por estar menos relacionado às mudanças de temperatura e aos níveis de estresse, melhorando o estado fisiológico destes $^{(7)}$.

Além dos benefícios destacados na revisão, também foi mencionado pelos enfermeiros que o banho enrolado promove outros efeitos benéficos no padrão fisiológico do bebê, ao reduzir choro, perda de peso, queda de saturação e apneia. Assim, o banho enrolado foi considerado, pelos participantes do estudo, como cuidado com benefícios diretos para o desenvolvimento de bebês prematuros, que extrapolam a higiene corporal em si, relacionados ao comportamento, conforto, relaxamento, estimulação sensoriomotora, segurança e estabilidade clínica. Tais resultados estão em conformidade com outras evidências internacionais que também recomendam o banho enrolado, pela associação à diminuição de estresse motor, choro e agitação, facilitando a interação e organização em bebês prematuros, em comparação ao banho convencional de imersão $0^{(7,9,12)}$.

Nessa lógica, compreende-se que o banho enrolado está em consonância com as diretrizes de cuidado humanizado ao recém-nascido do Ministério da Saúde brasileiro que reforça a necessidade de respeito ao estado comportamental do bebê para realização e uso de enrolamento para segurança no contato com a água. Ademais, sinaliza que o banho não deve ser procedimento estressante e, no escopo dos cuidados neuroprotetores, deve envolver medidas que reduzam o estresse térmico e comportamental ${ }^{(13)}$, justamente o que o banho enrolado minimiza, conforme os achados atuais.

Além da estabilidade clínica e peso, destacados pelos enfermeiros, a literatura aponta que a idade gestacional corrigida também pode ajudar o profissional quanto à decisão da realização do banho, no entanto, o profissional deve estar atento às necessidades singulares de cada bebê $\hat{1}^{(14)}$, sendo necessário reduzir os potenciais estressores no ambiente da unidade neonatal, como ruídos e iluminação excessiva. Além disso, a preocupação com a prevenção de estresse térmico também constou nos achados desta pesquisa. A esse respeito, a literatura enfatiza a necessidade de mais estudos de banhos de imersão, pois, ainda, não há consenso sobre a redução do estresse térmico com enrolamento ${ }^{(6,15)}$.

Outro resultado importante foi a oportunidade de interação tranquila entre bebê-família-profissional nessa modalidade de banho. Nessa perspectiva, a literatura aponta os benefícios desse cuidado para os pais, pois proporciona maior confiança em suas funções, melhor interação com a criança e diminuição do estresse parental. Assim, esse estímulo ao envolvimento dos pais no banho fornece a eles segurança para continuidade do cuidado com o bebê prematuro, na volta para casa ${ }^{(13)}$.

Foram mencionados pelos enfermeiros fatores que influenciaram negativamente na prática do banho na unidade neonatal, sendo estes: desconhecimento, baixa adesão e sobrecarga de trabalho da equipe de enfermagem, devido à escassez de recursos humanos, ausência de rotinas e protocolos institucionais, além da carência ou mesmo inadequação dos recursos materiais disponíveis nas instituições. Evidências indicam que esses aspectos afetam de forma negativa a saúde do trabalhador, provocando descontentamento, sobrecarga física e psíquica, absenteísmo e estresse. Ademais, prejudicam, também, a qualidade da assistência ao bebê e à família, dificultando ou ainda im- 
possibilitando a realização de cuidados ideais e boas práticas $^{(16-17)}$.

Os enfermeiros sinalizaram nesta pesquisa a existência, em algumas instituições, apenas de protocolos para o banho de imersão convencional e, portanto, expressaram a necessidade do estabelecimento de protocolo na unidade, para instaurar rotina para realização do banho enrolado. Contudo, há recomendações que indicam a necessidade de inclusão da modalidade de enrolamento nos protocolos de banho em unidades neonatais para bebês a termo e prematuros tardios que estejam estáveis clinicamente, além do desenvolvimento de ações educativas de treinamento das equipes para aquisição de atitudes que minimizem estresse neonatal ${ }^{(14,18)}$, o que também foi constatado nas falas dos participantes.

Portanto, os protocolos devem contemplar as reais necessidades dos serviços e do grupo populacional que será assistido, ao funcionarem como instrumentos guias para o enfermeiro, pois sistematizam a assistência e padronizam o atendimento na instituição, garantindo a autonomia e segurança para os profissionais, pois norteiam o cuidado a ser prestado ${ }^{(19)}$, que é premente, no que tange à prática do banho enrolado para garantir segurança aos bebês, familiares e profissionais de enfermagem.

Cumpre enfatizar que a equipe de enfermagem tem papel determinante na concretização da atenção humanizada ao recém-nascido de risco, por meio do acolhimento da família e dos cuidados individualizados que favoreçam o desenvolvimento dos bebês e a segurança e adaptação de familiares durante a hospitalização. Porém, para melhorar a adesão dos profissionais às boas práticas, inclusive relacionadas ao banho, é preciso reconhecer, no contexto da Unidade Neonatal, as fortes influências de questões gerenciais, como recursos humanos, interação entre equipes, processos de trabalho, estratégias de liderança e gerenciamento do cuidado ${ }^{(20)}$.

Ademais, para melhores práticas no banho do bebê prematuro, os enfermeiros ressaltaram a necessidade de maior engajamento, sensibilização e disseminação do conhecimento sobre esse tipo de banho.
Sabe-se que a prática de enfermagem necessita estar fundamentada em conceitos e reflexões científicas, demandando integrar educação e trabalho. Por meio da capacitação, as equipes podem apreender conhecimentos sobre cuidados desenvolvimentais que possibilitem melhor modulação do manuseio, de modo a respeitar as necessidades do bebê prematuro, uma vez que o comportamento do profissional também está intimamente relacionado ao estresse sofrido pelo prematuro. Logo, a literatura recomenda que enfermeiros e equipes sejam treinados para oferecer comportamento protetivo, durante o manuseio, no banho do bebê prematuro ${ }^{(14)}$, e o banho enrolado se apresenta como cuidado humanizado, capaz de alcançar esse intuito.

\section{Conclusão}

Na perspectiva dos enfermeiros participantes, o banho enrolado tem efeitos positivos no relaxamento, na estabilidade clínica e no desenvolvimento de bebês prematuros, além de promover segurança destes e dos respectivos pais, porém precisam estar clinicamente estáveis, para que a técnica humanizada de cuidado seja aplicada. No entanto, existem desafios gerenciais para efetiva implementação, que incluem a excessiva carga de trabalho, a carência de insumos materiais, o desconhecimento por parte dos profissionais. Logo, é uma prática que precisa ser disseminada e fortalecida, através de ações gerenciais efetivas, em unidades neonatais.

\section{Colaborações}

Santos HM contribuiu na concepção do projeto, coleta, análise, interpretação dos dados e redação do artigo. Silva LJ colaborou com concepção do projeto, análise, interpretação dos dados, redação do artigo, revisão crítica do conteúdo intelectual e aprovação final da versão a ser publicada. Santos ACN auxiliou na concepção do projeto. Góes FGB, Araújo BBM e Santos IMM contribuíram na redação do artigo e revisão crítica relevante do conteúdo intelectual. 


\section{Referências}

1. Costa MFS, Gomes Jr SC, Magluta C. Analysis of the distribution of births with severity markers in maternity with neonatal intensive care unit in the Unified Health System. Cad Saúde Coletiva. 2018; 26(2):125-30. doi: http://dx.doi. org/10.1590/1414-462x201800020419

2. Pessoa TA, Martins CBG, Lima FCA, Gaíva MAM. The growth and development against the prematurity and low birth weight. Av Enferm [Internet]. 2015 [cited may 28, 2019]; 33(3):401-11. Available from: http://www.scielo.org.co/pdf/aven/ v33n3/v33n3a08.pdf

3. Ministério da Saúde (BR). Atenção humanizada ao recém-nascido de baixo peso. Método Canguru: manual técnico [Internet]. 2017 [citado 2019 out 22]. Disponível em: http://bvsms.saude.gov.br/ bvs/publicacoes/atencao_humanizada_metodo_ canguru_manual_3ed.pdf

4. PhillipsRM.Seven coremeasures ofneuroprotective family-centered developmental care: creating an infrastructure for implementation. Newborn Infant Nurs Rev 2015; 15:87-90. doi: https://doi. org/10.1053/j.nainr.2015.06.004

5. Marques LF, Ribeiro RV, Rocha CR, Carreiro MA, Santiago LC. Care to the extreme premature: minimum handling and humanization. Rev Pesqui Cuid Fundam Online. 2017; 9(4):927-31. doi: http://dx.doi.org/10.9789/2175-5361.2017. v9i4.927-931

6. Freitas $\mathrm{P}$, Munhoz MMB, Costa P, Kimura AF. Effect of two immersion bathing techniques on the axillary temperature of preterm newborns: a pilot study. Texto Contexto Enferm. 2018; 27(1):e0580016. doi: http://dx.doi. org/10.1590/0104-070720180000580016

7. Fernández D, Antolín-Rodriguéz R. Bathing a premature infant in the intensive care unit: a systematic review. J Pediatr Nurs. 2018; 42:e52-e57. doi: https://doi.org/10.1016/j.pedn.2018.05.002

8. Freitas P, Bueno M, Holditch-Davis D, Santos Jr HP, Kimura AF. Biobehavioral responses of preterm infants to conventional and swaddled tub baths. J Perinat Neonat Nurs. 2018; 32(4):358-65. doi: http://dx.doi.org/10.1097/ JPN.0000000000000336
9. Çaka SY, Gözen D. Effects of swaddled and traditional tub bathing methods on crying and physiological responses of newborns. J Spec Pediatr Nurs. 2018; 23(1):e12202. doi: https:// doi-org.ez39.periodicos.capes.gov.br/10.1111/ jspn.12202

10. Vinuto J. A amostragem em bola de neve na pesquisa qualitativa: um debate em aberto. Temáticas [Internet]. 2014 [citado 2019 mar. 14];22(4):20320. Disponível em: https://pdfs.semanticscholar. org/cd8e/3ecb215bf9ea6468624149a343f8a1fa8456.pdf

11. Oliveira, DC. Análise de conteúdo temáticocategorial: uma proposta de sistematização. Rev Enferm UERJ [Internet]. 2008 [citado 2018 mar. 2]; 16(4):569-76. Disponível em: http://www. facenf.uerj.br/v16n4/v16n4a19.pdf

12. Edraki M, Paran M, Montaseri S, Nejadi MR, Montaseri Z. Comparing the effects of swaddled and conventional bathing methods on body temperature and crying duration in premature infants: a randomized clinical trial. J Caring Sci. 2014;3(2):83-91.doi: http://dx.doi.org/10.5681/ jcs.2014.009

13. Ministério da Saúde (BR). Método canguru: diretrizes do cuidado [Internet]. 2018 [citado 2019 out 22]. Disponível em: http://portaldeboaspraticas.iff.fiocruz.br/wp-content/uploads/2018/09/ metodo_canguru_diretrizes_cuidado2018.pdf

14. Lund C. Bathing and beyond: current bathing controversies for newborn infants. Adv Neonatal Care. 2016; 16(5):13-20. doi: http://dx.doi. org/10.1097/ANC.0000000000000336

15. Freitas P, Marques SR, Alves TB, Takahashi J, Kimura AF. Changes in physiological and behavioral parameters of preterm infants undergoing body hygiene: a systematic review Rev Esc Enferm USP. 2014; 48(spe):178-83. doi: http://dx.doi. org/10.1590/S0080-623420140000600025

16. Trettene A, Luiz A, Razera A, Maximiano T, Cintra F, Monteiro L. Nursing workload in specialized semi-intensive therapy unit: workforce size criteria. Rev Esc Enferm USP. 2015; 49(6):95863. doi: http://dx.doi.org/10.1590/S0080623420150000600012

17. Lima LSC, Souza NVDO, Gonçalves FGA, Pires AS, Ribeiro LV, Santos DM. Subjectivity of the nursing 
workforce and the practice of adapting and improvising material. Cienc Cuid Saúde. 2016; 15(4):685-92. doi: https://doi.org/10.4025/ cienccuidsaude.v15i3.31555

18. Brandon D, Hill CM, Heimall L, Lund CH, Kuller J, McEwan T, et al. Neonatal skin care: evidencebased clinical practice guideline. [Internet]. 201 [cited Dec 13, 2019]. Available from: https:// research-portal.uws.ac.uk/en/publications/ neonatal-skin-care-evidence-based-clinicalpratice-guideline
19. Amaral GF, Sales ASD, Mota PMT, Monteiro SBLL, Cordeiro SPSR, Cavalcante MM. Development of a nursing protocol for childcare consultations. Rev Rene. 2015; 16(1):81-9. doi: http://dx.doi. org/10.15253/2175-6783.2015000100011

20. Silva LJ, Leite JL, Silva TP, Silva IR, Mourão PP, Gomes TM. Management challenges for best practices of the Kangaroo Method in the Neonatal ICU. Rev Bras Enferm. 2018; 71(Suppl 6):278391. doi: http://dx.doi.org/10.1590/0034-71672018-0428 